\title{
A microbiological inhibition method for the rapid, broad-spectrum, and high-throughput screening of 34 antibiotic residues in milk
}

\author{
Qin Wu, ${ }^{1}$ Qiang Zhu, ${ }^{1}$ Yana Liu, ${ }^{1}$ Muhammad Abu Bakr Shabbir, ${ }^{1}$ Adeel Sattar, ${ }^{2}$ Dapeng Peng, ${ }^{3}$ Yanfei Tao, ${ }^{3}$ \\ Dongmei Chen, ${ }^{3}$ Yulian Wang, ${ }^{3 *}$ and Zonghui Yuan ${ }^{3 *}$ \\ ${ }^{1}$ National Reference Laboratory of Veterinary Drug Residues and MOA Key Laboratory for Detection of Veterinary Drug Residues, \\ Huazhong Agricultural University, Wuhan, Hubei 430070, China \\ ${ }^{2}$ Department of Pharmacology and Toxicology, University of Veterinary and Animal Sciences, Lahore 54000, Pakistan \\ ${ }^{3}$ MOA Laboratory for Risk Assessment of Quality and Safety of Livestock and Poultry Products, Huazhong Agricultural University, Wuhan, \\ Hubei 430070, China
}

\section{ABSTRACT}

In this study, we developed a microbiological inhibition method for the rapid screening of antibiotics in milk with Geobacillus stearothermophilus ATCC12980 as an indicator bacterium and an easy sample pretreatment. We observed that the limits of detection of the kit for 34 common antibiotic residues in milk, including $\beta$-lactams (13), aminoglycosides (6), tetracyclines (4), sulfonamides (6), macrolides (4), lincosamides (1), were lower than or close to the maximum residue limits formulated by the European Union and China. Moreover, the false-positive rate was $1 \%$ and the falsenegative rates were less than $5 \%$. The ruggedness of the method (the reproducibility of detection capability of different batches of medium) met requirements at determined levels and residual limits. The shelf life of the kit was more than 6 mo at $4^{\circ} \mathrm{C}$. Additionally, we observed good correlations between the kit results and ultra-high-performance liquid chromatography-tandem mass spectrometry results for incurred milk (samples taken from animals treated with antibiotics according to the pre-slaughter medication data), which indicated that the kit was reliable for screening antibiotics in incurred samples. In conclusion, the kit has a broad application potential with high sensitivity, specificity, and reproducibility, stability, and reliability, combined with simple operation, low cost, and high-throughput capacity.

Key words: antibiotic residue, microbiological inhibition method, Geobacillus stearothermophilus ATCC12980, milk

\footnotetext{
Received February 13, 2019.

Accepted July 16, 2019.

*Corresponding authors: wangyulian@mail.hzau.edu.cn and yuan5802@mail.hzau.edu.cn
}

\section{INTRODUCTION}

Antimicrobial agents are widely used for the control and treatment of cow mastitis, laminitis, and other infections caused by bacteria (Baur et al., 2017; Breen et al., 2017). Long-term or irregular usage of antibiotics can lead to residues in raw milk and dairy products. Antibiotic residues in milk may cause allergic reactions and even interfere with intestinal flora and the development of resistance to antibiotics, which is harmful to human health. Moreover, antibiotic residues cause serious economic losses to the food processing industry because they interfere with the fermentation of cheese and yogurt (Darwish et al., 2013; Zeina et al., 2013; de Albuquerque Fernandes et al., 2014; Tumini et al., 2015; Berruga et al., 2016). Accordingly, it is very important to control the residues of antimicrobial drugs in milk.

For these reasons, several control authorities, such as the European Union (Regulation EC/37/2010; European Union, 2010) and Codex (FAO/WHO, 2015), have determined maximum residue limits (MRL) for specified veterinary drugs in animal food, including milk. Several physicochemical methods have been developed for the screening and confirmation of residues of antimicrobial agents in milk, such as HPLC with UV (Choma et al., 2012; Chu et al., 2017). These methods are highly sensitive, selective, and reliable. Moreover, the development of mass spectrometry has further improved the sensitivity of physicochemical methods. Additional separation and analysis technologies can be coupled to tandem MS, such as gas chromatography (GC-MS/MS; Steinborn et al., 2016) or liquid chromatography-tandem MS (LC-MS/MS; Tian et al., 2016; Wang et al., 2016; Jank et al., 2017). Nevertheless, these physicochemical methods require expensive equipment, highly trained personnel, and sophisticated sample preparation. Immunological methods, such as receptor methods (Mor et al., 2012) and ELISA (Tao et al., 2014), are widely used for the detection of anti- 
biotics because they are sensitive, reliable, rapid, and portable; however, they are only sensitive to a group of antibiotics or even specific antibiotics.

Microbiological inhibition methods have been extensively used for food safety monitoring because of their reliability, usability, portability, simplicity of operation, low cost, broad spectrum, and high-throughput capability, along with other benefits (Ezenduka et al., 2014; Hakimzadegan et al., 2014; Mata et al., 2014; Beltrán et al., 2015). Several well-established kits have been developed for microbiological inhibition methods based on test tubes for rapidly screening antibiotics in milk. These include the brilliant black reduction test (BRT AiM, AiM-Analytik in Milch Produktions-und Vertriebs GmbH, München, Germany; Molina et al., 2003), Copan milk test (Copan Italia SpA, Brescia, Italy; Le Breton et al., 2007), Delvotest SP-NT (DSM Food Specialties, Delft, the Netherlands; Althaus et al., 2003a), Eclipse 100 (ZEU-Inmunotec SL, Zaragoza, Spain; Beltrán et al., 2015), and Charm Blue-Yellow II (Charm Sciences, Lawrence, MA; Linage et al., 2007). Nevertheless, no ideal microbiological inhibition method exists for the detection of all types of common antibiotics used in milk, including $\beta$-lactams, aminoglycosides, tetracyclines, macrolides, sulfonamides, and lincosamides. Moreover, milk can interfere with the detection capability of microbiological inhibition methods and cause false results because of its matrix effects. Some researchers improved sample pretreatment methods using the prediffusion process and solvent extraction approach (Molina et al., 2003; Stead et al., 2004; Montero et al., 2005). However, both of these sample pretreatment methods are laborious and time consuming. Furthermore, the solvent extraction approach can only be used in the laboratory, not in the field or on the farm.

In this study, we developed a microbiological inhibition method for the rapid detection of 6 classes of antibiotics: $\beta$-lactams, aminoglycosides, tetracyclines, macrolides, sulfonamides, and lincosamides in milk. We also developed a simple sample preparation method and used Geobacillus stearothermophilus ATCC12980 as an indicator bacterium.

\section{MATERIALS AND METHODS}

\section{Chemicals and Standard Solutions}

The following antibiotics were purchased from SigmaAldrich (St. Louis, MO): $\beta$-lactams: ampicillin (AMP), penicillin G (PENG), amoxicillin (AMO), cloxacillin (CLO), dicloxacillin (DIC), penicillin V (PENV), oxacillin (OXA), nafcillin (NAP), ceftiofur (CEFT), cefuroxime (CEFM), cefalexin (CEFA), cefoperazone (CEFZ), and cefazolin (CEFL); tetracyclines: doxycycline (DOX), tetracycline (TET), oxytetracycline (OXY), and chlortetracycline (CTE); aminoglycosides: streptomycin (STR), neomycin (NEO), dihydrostreptomycin (DSTR), gentamicin (GEN), kanamycin (KAN), and spectinomycin (SPE); sulfonamides: sulfamonomethoxine (SMM), sulfadimidine (SDM), sulfadiazine (SDZ), sulfamethoxypyridazine (SMP), sulfaquinoxaline (SQX), and sulfamethoxazole (SMX); macrolides: erythromycin (ERY), tylosin (TYL), tilmicosin (TIL), spiramycin (SPI); and lincosamides: lincomycin (LIN), trimethoprim (TMP), and chloramphenicol (CAP).

The components used in the preparation of stock solutions and working solutions of antibiotics are shown in Table 1. Stock solutions of antimicrobial agents (1 $\mathrm{mg} / \mathrm{mL}$ ) were prepared in suitable solvents and stored at $-20^{\circ} \mathrm{C}$ in amber glass vials for up to $2 \mathrm{wk}$. Working standard solutions $(1 \mu \mathrm{g} / \mathrm{mL})$ were prepared by suitable dilutions of the stock solutions until use.

\section{Preparation of Test Organism Spore Suspensions}

Geobacillus stearothermophilus ATCC12980 was obtained from the American Type Culture Collection (Manassas, VA). The sporulation medium used for the production of $G$. stearothermophilus ATCC12980 spores contained $7.0 \mathrm{~g}$ of tryptone, $6.0 \mathrm{~g}$ of peptone,

Table 1. Methods for the preparation of stock solutions and working standard solutions of antibiotics

\begin{tabular}{lll}
\hline Antimicrobial agent & Solvent & Diluent \\
\hline$\beta$-Lactams & Phosphate buffer, pH 6.0,0.1 mol/L & Phosphate buffer, pH 6.0, 0.1 mol/L \\
Tetracyclines & HCl, 0.1 mol/L & Phosphate buffer, pH 6.0, 0.1 mol/L \\
Aminoglycosides & Tris, pH 8.0,0.01 mol/L & Tris, pH 8.0, 0.01 mol/L \\
Sulfonamides & NaOH, 0.1 mol/L & Sterilized distilled water \\
Macrolides & Phosphate buffer, pH 8.0,0.01 mol/L & Phosphate buffer, pH 8.0, 0.01 mol/L \\
Lincosamides & Phosphate buffer, pH 8.0,0.01 mol/L & Phosphate buffer, pH 8.0, 0.01 mol/L \\
Trimethoprim & Glacial acetic acid & Sterilized distilled water \\
Chloramphenicol & Methanol & Sterilized distilled water \\
\hline
\end{tabular}


$3.0 \mathrm{~g}$ of yeast powder, $1.5 \mathrm{~g}$ of beef extract, $0.03 \mathrm{~g}$ of $\mathrm{MnSO}_{4} \cdot \mathrm{H}_{2} \mathrm{O}, 0.06 \mathrm{~g}$ of $\mathrm{MgSO}_{4}, 0.04 \mathrm{~g}$ of $\mathrm{CaCl}_{2}, 0.05$ $\mathrm{g}$ of $\mathrm{KH}_{2} \mathrm{PO}_{4}$, and $1,000 \mathrm{~mL}$ of distilled water; it was sterilized at $121^{\circ} \mathrm{C}$ for 15 min before use.

To prepare the test-organism spore suspension, $G$. stearothermophilus ATCC12980 in logarithmic phase (9 to $12 \mathrm{~h}$ ) was aseptically inoculated into sporulation medium and cultured at a constant temperature at $55^{\circ} \mathrm{C}$ for $72 \mathrm{~h}$ with oscillation at $220 \mathrm{rpm}$. The cells were then harvested by centrifugation at $3,600 \times g$ and $4^{\circ} \mathrm{C}$ for $20 \mathrm{~min}$. The supernatant was decanted and the cells were resuspended in sterile physiological saline $(0.85 \% \mathrm{NaCl})$. This step was carried out twice. Then, the cells were suspended in sterile physiological saline and heated in water bath at $80^{\circ} \mathrm{C}$ for 20 min to kill the remaining vegetative cells and promote the formation of spores. The concentration of the spore suspension was adjusted to approximately $5 \times 10^{10} \mathrm{cfu} / \mathrm{mL}$, as determined by the plate count method and incubation at $55^{\circ} \mathrm{C}$ for $24 \mathrm{~h}$. Finally, aliquots of spore suspensions were stored at $4^{\circ} \mathrm{C}$ until use.

\section{Preparation of Test Medium}

The test kit's medium contained $3.0 \mathrm{~g}$ of beef extract, $5.0 \mathrm{~g}$ of peptone, $1.7 \mathrm{~g}$ of enzymatic hydrolysis of casein peptone, $0.3 \mathrm{~g}$ of soy peptone, $0.25 \mathrm{~g}$ of $\mathrm{K}_{2} \mathrm{HPO}_{4}, 0.5 \mathrm{~g}$ of $\mathrm{NaCl}, 5.25 \mathrm{~g}$ of glucose, $30 \mathrm{~g}$ of polyethylene glycol 4000, $7 \mathrm{~g}$ of sodium carboxymethylcellulose, $0.003 \mathrm{~g}$ of $\mathrm{MnSO}_{4} \cdot \mathrm{H}_{2} \mathrm{O}, 0.006 \mathrm{~g}$ of $\mathrm{MgSO}_{4}, 0.004 \mathrm{~g}$ of $\mathrm{CaCl}_{2}$, $15.0 \mathrm{~g}$ of agar, and 1,000 mL of distilled water. The dissolved medium was autoclaved at $121^{\circ} \mathrm{C}$ for $15 \mathrm{~min}$. After the medium was cooled to $50 \pm 1^{\circ} \mathrm{C}$, the $\mathrm{pH}$ was adjusted to $7.8 \pm 0.1$. Once prepared, G. stearothermophilus ATCC12980 spore suspension $\left(3 \times 10^{7} \mathrm{cfu} / \mathrm{L}\right)$ along with $0.1 \mathrm{mg} / \mathrm{L}$ bromocresol purple indicator (Mallinckrodt, Staines, UK), $50 \mu \mathrm{g} / \mathrm{L}$ TMP, and 200 $\mu \mathrm{g} / \mathrm{L}$ CAP were added. Then, $150 \mu \mathrm{L}$ of the culture medium was added into each well of microtiter plates using an electronic pipette (Eppendorf Research Pro, Eppendorf, Hamburg, Germany) after the kit's medium components were well mixed by vortexing. Finally, these microtiter plates were sealed with aluminum foil and stored at $4^{\circ} \mathrm{C}$ until use.

\section{Preparation of Milk Samples}

Blank Milk Samples. Milk samples were collected from postpartum Holstein cows (between 60 and $90 \mathrm{~d}$ postpartum; Debayle et al., 2008) with a history of no antibiotics administered during the previous 9 wk at the dairy farm of Huazhong Agricultural University (Wuhan, Hubei, China). Milk samples were kept at $4^{\circ} \mathrm{C}$ for approximately $2 \mathrm{~d}$ throughout the experiment.
Spiked Milk Samples. Spiked samples were prepared by adding working standard solutions of the respective antibiotics in a single step to antibiotic-free milk at the spiked levels given in Table 2.

\section{Microbiological Inhibition Test and Interpretation of Results}

The microtiter wells needed were cut off and the aluminum foil was removed carefully. First, the blank and spiked milk samples were incubated in a water bath for $10 \mathrm{~min}$ at $80^{\circ} \mathrm{C}$. Second, $50 \mu \mathrm{L}$ of control and spiked milk samples were added into each well of the microtiter plate, respectively, and the wells were sealed firmly with the plastic foil supplied with the kit. Finally, wells with milk samples were incubated in a water bath or incubator at $65^{\circ} \mathrm{C}$ until the wells with blank samples had turned yellow (approximately 2.5 to $3 \mathrm{~h}$ ). At the end of incubation, the detection results were decided based on the color change of the microtiter wells. An example is shown in Figure 1: yellow indicates a negative result; half yellow, half purple indicates limit of detection; and purple indicates a positive result. When the color change of the microtiter was between purple and half yellow/half purple, the result was questionable; we regarded these results as positive.

\section{Validation Protocol}

Limit of Detection. Dose-response curves of these drugs were established according to the ISO13969:2003 guidelines (ISO, 2003). For each drug, 8 concentrations were prepared, with 20 replicates for each concentration on each of 5 different days. Limits of detection (LOD) were determined as the concentration that resulted in 95\% positive results for the lowest concentration (ISO 13969:2003; ISO, 2003). In addition, LOD were determined using only one batch of medium.

Specificity and Selectivity. For determination of the false-positive rate, 100 blank milk samples were analyzed using the kit in the present study. The falsepositive rate was estimated as follows:

\section{False-positive rate $=$}

(no. of positive samples/total blank samples) $\times 100 \%$.

Generally, the false-negative rate of a screening method focuses on samples spiked at concentrations of the determined level (LOD) and residual limit (MRL), which should be not more than 5\%. The determined LOD met the true detection capability of the method when the false-negative rate of LOD was $\leq 5 \%$. Moreover, the method is reliable for screening positive samples at 
Table 2. The detection capability of the kit for different antibiotics in milk $(\mu \mathrm{g} / \mathrm{L})$

\begin{tabular}{|c|c|c|c|c|c|}
\hline Antibiotic & Spiked level $(\cdot / \mathrm{L})$ & \multicolumn{2}{|c|}{ MRL1 } & $\begin{array}{c}\text { LOD2 } \\
(\cdot / \mathrm{L})\end{array}$ & $\begin{array}{l}\text { Range of dose- } \\
\text { response curse }\end{array}$ \\
\hline Penicillin G & $0,1,2,3,4,5,6,8$ & 4 & 4 & 2 & $1-3$ \\
\hline Amoxicillin & $0,1,2,3,4,5,6,8$ & 10 & 4 & 3 & $1-3$ \\
\hline Cloxacillin & $0,2.5,5,10,15,20,30,40$ & 30 & 30 & 20 & $10-20$ \\
\hline Dicloxacillin & $0,2.5,5,10,15,20,30,40$ & 30 & 30 & 20 & $10-20$ \\
\hline Ceftiofur & $0,2.5,5,10,20,40,60,80$ & 100 & 100 & 80 & $20-60$ \\
\hline Cefalexin & $0,2.5,5,10,20,40,60,80$ & 100 & 100 & 20 & $5-20$ \\
\hline Cefoperazone & $0,2.5,5,10,20,40,60,80$ & 50 & 50 & 50 & $10-40$ \\
\hline Cefazolin & $0,2.5,5,10,20,40,60,80$ & 50 & 50 & 20 & $5-20$ \\
\hline Cefuroxime & $0,2.5,5,10,20,40,60,80$ & 50 & - & 50 & $10-40$ \\
\hline Streptomycin & $0,50,100,200,250,500,750,1,000$ & 200 & 200 & 500 & $200-500$ \\
\hline Doxycycline & $0,25,50,75,100,200,300,400$ & - & - & 200 & $50-100$ \\
\hline Chlortetracycline & $0,25,50,75,100,200,300,400$ & 100 & 100 & 200 & $100-300$ \\
\hline Tetracycline & $0,25,50,75,100,200,300,400$ & 100 & 100 & 200 & $200-300$ \\
\hline Oxytetracycline & $0,25,50,75,100,200,300,400$ & 100 & 100 & 200 & $200-300$ \\
\hline Erythromycin & $0,10,20,30,40,50,75,100$ & 40 & 40 & 50 & $30-50$ \\
\hline Tylosin & $0,10,20,30,40,50,75,100$ & 50 & 50 & 50 & $30-50$ \\
\hline Tilmicosin & $0,10,20,30,40,50,75,100$ & 50 & 50 & 50 & $30-50$ \\
\hline Spiramycin & $0,400,500,600,700,800,1,200,1,600$ & 200 & 200 & 700 & $600-800$ \\
\hline Sulfadimidine & $0,25,50,75,100,150,200,300$ & 25 & 100 & 150 & $75-150$ \\
\hline Sulfadiazine & $0,25,50,75,100,150,200,300$ & 100 & 100 & 75 & $50-100$ \\
\hline Sulfamethoxazole & $0,25,50,75,100,150,200,300$ & 100 & 100 & 50 & $25-75$ \\
\hline Sulfamonomethoxine & $0,25,50,75,100,150,200,300$ & 100 & 100 & 50 & $25-75$ \\
\hline Sulfamethoxypyridazine & $0,25,50,75,100,150,200,300$ & 100 & 100 & 50 & $25-75$ \\
\hline Sulfaquinoxaline & $0,25,50,75,100,150,200,300$ & 100 & 100 & 100 & $75-150$ \\
\hline
\end{tabular}

${ }^{1}$ Maxiumum residue limits of China and the European Union.

${ }^{2}$ Limit of detection.

${ }^{3}$ No MRL.

the MRL when the false-negative rate of the MRL was $\leq 5 \%$. Therefore, to determine the false-negative rate, 100 blank milk samples spiked at the MRL and LOD concentrations for each drug were analyzed using this kit. We calculated the false-negative rate as follows:

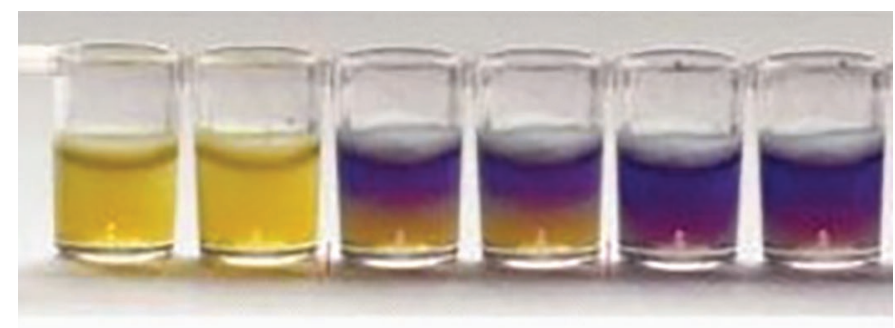

\section{Negative Detection limit Positive}

Figure 1. Yellow color indicates a negative result, half yellow/ half purple indicates limit of detection, and purple color indicates a positive result
False-negative rate $=$ (no. of negative samples/

total spiked samples) $\times 100 \%$.

Ruggedness. To determine the ruggedness of the kit, we evaluated the effects of different batches of the kit's medium on the sensitivity to all antibiotics. Furthermore, we assessed positive rates for antibiotics at the LOD, MRL in China, MRL in the European Union (EU), and 2 more concentrations around the LOD using 5 different batches of medium.

Comparison with Commercially Available Test Kits. Fifteen postpartum Holstein cows (between 60 and $90 \mathrm{~d}$ postpartum) and having no antibiotic exposure within the last $9 \mathrm{wk}$ were raised at the dairy farm of Huazhong Agricultural University (Wuhan). Fifteen cows were divided into 5 groups, and each group was treated with AMP $(20 \mathrm{mg} / \mathrm{kg})$, GEN $(4 \mathrm{mg} / \mathrm{kg})$, OXY $(20 \mathrm{mg} / \mathrm{kg})$, or SDZ $(30 \mathrm{mg} / \mathrm{kg})$ by intramuscular injection, or TYL $(13 \mathrm{mg} / \mathrm{kg})$ by intravenous injection. 
Milk samples were collected at intervals of $0,24,48,72$, and $96 \mathrm{~h}$ separately from each cow after drug administration. Finally, all collected samples were screened for the presence of antibiotics in milk and confirmed by the study kit, the Eclipse 50 test (ZEU-Inmunotec, Zaragoza, Spain), and ultra-high-performance liquid chromatography/tandem MS (UPLC-MS/MS; Tian et al., 2016).

Stability. The stability of the kit was determined based on appearance, smell, and performance, including detection capability and incubation time of 3 batches of kits stored at $4^{\circ} \mathrm{C}$ for 6 mo $(0,30,60,90,120,150$, and $180 \mathrm{~d}$ ). The appearance and smell of the kit can be observed by visual and olfactory senses. Detection capability and incubation time can be determined according to the process described in the "Microbiological Inhibition Test and Interpretation of Results" section. The stability of the detection capability of the kit focused on 5 representative antibiotics: AMP $(2 \mu \mathrm{g} / \mathrm{L})$, GEN $(50 \mu \mathrm{g} / \mathrm{L})$, OXY $(100 \mu \mathrm{g} / \mathrm{L})$, TYL $(50 \mu \mathrm{g} / \mathrm{L})$, and SDZ $(50 \mu \mathrm{g} / \mathrm{L})$, representing 5 different groups of antibiotics.

\section{RESULTS}

\section{Detection Capability}

The detection capability of the kit in the present study for different antibiotics in milk is shown in Table 2. The LOD of the kit for $\beta$-lactams, aminoglycosides (GEN, NEO), macrolides (TYL, TIL), and sulfonamides were less than or equal to the MRL determined by China and the EU. However, the LOD for tetracyclines, STR, DSTR, KAN, SPE, ERY, SPI, SDM, and LIN were higher than the MRL for China and the EU.

\section{Specificity and Selectivity}

Results of the false-negative rates of the kit in the present study for each antibiotic in milk are shown in Table 3. The false-negative rates of the kit for 34 antibiotics detected in this study were less than $5 \%$ at the determined levels and MRL. Therefore, the false-negative rates of the kit met the ISO13969:2003 guidelines (ISO, 2003). The false-positive rate of the kit was $1 \%$.

\section{Ruggedness}

The positive results of antibiotics at the LOD, China MRL, EU MRL, and 2 more concentrations around the LOD evaluated using 5 batches of kit medium are given in Table 4 . The positive rate of spiked samples at concentrations below the LOD was less than $95 \%$ for some batches of medium and some antibiotics. Nonetheless, the positive rate of spiked samples at the LOD were $\geq 95 \%$. Therefore, the determined LOD met the true detection capability of the method. In addition, the positive rates of spiked samples at the China and EU MRL were $>95 \%$ when the LOD were lower than the MRL for most antibiotics. Nevertheless, the positive rate of spiked samples at the MRL was $<95 \%$ for a few antibiotics such as streptomycin and dihydrostreptomycin, for which the LOD were higher than the MRL. Therefore, the present study was reliable for screening positive samples at the MRL for most antibiotics in milk. The detection capability of the present kit needs to be improved for some antibiotics such as streptomycin and dihydrostreptomycin.

\section{Comparison with Commercially Available Test Kits and Confirmation by UPLC/MS-MS}

The results of the screening and quantification of different groups of antibiotics in incurred milk (samples taken from animals treated with antibiotics according to the pre-slaughter medication data) by the test kit, the Eclipse 50 kit, and by UPLC/MS-MS are shown in Table 5. Screening results of 5 antibiotics in milk by the test kit and Eclipse 50 were similar; 18 positive samples were detected by the test kit and 15 positive samples were detected by the Eclipse 50 kit. Additionally, no false-positive results were detected after confirmation by UPLC-MS/MS. However, negative samples (as determined by the kit) contained antimicrobial residues at concentrations lower than the LOD of the test kit after UPLC-MS/MS confirmation. Because UPLCMS/MS using a solvent extraction method for sample preparation is more sensitive to all groups of antibiotics than the kit used in present study, the kit is reliable to screen antibiotics in actual samples.

\section{Stability}

The stability of the kit was determined based on appearance, smell, detection time, and detection capability of the kit. We have provided more information about how to determine these parameters in the "Materials and Methods: Stability" section. Results indicated that all parameters of this kit did not change over 6 mo at $4^{\circ} \mathrm{C}$. Consequently, the shelf life of the kit in this study is more than 6 mo at $4^{\circ} \mathrm{C}$.

\section{DISCUSSION}

\section{Detection Capability}

Geobacillus stearothermophilus is sensitive to penicillins and cephalosporins but insensitive to other 
WU ET AL.

Table 3. Results of the false-negative rates of the kit tested in 100 samples

\begin{tabular}{|c|c|c|c|c|c|c|}
\hline \multirow[b]{2}{*}{ Antibiotic } & \multicolumn{3}{|c|}{ Spiked level $^{1}(\cdot / \mathrm{L})$} & \multicolumn{3}{|c|}{ False-negative rate $(\%)$} \\
\hline & MRL (China) & MRL (EU) & LOD & MRL (China) & MRL (EU) & LOD \\
\hline Penicillin G & 4 & 4 & 2 & 0 & 0 & 3 \\
\hline Ampicillin & 10 & 4 & 3 & 0 & 0 & 0 \\
\hline Amoxicillin & 10 & 4 & 3 & 0 & 0 & 0 \\
\hline Cloxacillin & 30 & 30 & 20 & 0 & 0 & 0 \\
\hline Dicloxacillin & 30 & 30 & 20 & 0 & 0 & 0 \\
\hline Oxacillin & 30 & 30 & 20 & 0 & 0 & 0 \\
\hline Naphthoxicillin & 30 & 30 & 9 & 0 & 0 & 0 \\
\hline Penicillin V & 30 & $-^{2}$ & 5 & 0 & $\mathrm{NG}^{3}$ & 2 \\
\hline Ceftiofur & 100 & 100 & 80 & 0 & 0 & 0 \\
\hline Cefalexin & 100 & 100 & 20 & 0 & 0 & 3 \\
\hline Cefoperazone & 50 & 50 & 50 & 0 & 0 & 0 \\
\hline Cefazolin & 50 & 50 & 20 & 0 & 0 & 0 \\
\hline Cefuroxime & 50 & - & 50 & 0 & NG & 0 \\
\hline Streptomycin & 200 & 200 & 500 & NG & NG & 0 \\
\hline Dihydrostreptomycin & 200 & 200 & 500 & NG & NG & 0 \\
\hline Gentamicin & 200 & 100 & 75 & 0 & 0 & 0 \\
\hline Neomycin & 500 & 1,500 & 75 & 0 & 0 & 0 \\
\hline Kanamycin & 200 & 150 & 1,250 & NG & NG & 0 \\
\hline Spectinomycin & 200 & 200 & 1,250 & NG & NG & 0 \\
\hline Doxycycline & - & - & 200 & NG & NG & 0 \\
\hline Chlortetracycline & 100 & 100 & 200 & 0 & 0 & 0 \\
\hline Tetracycline & 100 & 100 & 200 & 0 & 0 & 0 \\
\hline Oxytetracycline & 100 & 100 & 200 & 0 & 0 & 0 \\
\hline Erythromycin & 40 & 40 & 50 & 0 & 0 & 0 \\
\hline Tylosin & 50 & 50 & 50 & 0 & 0 & 0 \\
\hline Tilmicosin & 50 & 50 & 50 & 0 & 0 & 0 \\
\hline Spiramycin & 200 & 200 & 700 & NG & NG & 0 \\
\hline Sulfadimidine & 25 & 100 & 150 & NG & 0 & 0 \\
\hline Sulfadiazine & 100 & 100 & 75 & 0 & 0 & 0 \\
\hline Sulfamethoxazole & 100 & 100 & 50 & 0 & 0 & 4 \\
\hline Sulfamonomethoxine & 100 & 100 & 50 & 0 & 0 & 3 \\
\hline Sulfamethoxypyridazine & 100 & 100 & 50 & 0 & 0 & 1 \\
\hline Sulfaquinoxaline & 100 & 100 & 100 & 0 & 0 & 0 \\
\hline Lincomycin & 150 & 150 & 300 & NG & NG & 0 \\
\hline
\end{tabular}

${ }^{1}$ Maxiumum residue limits (MRL) of China and the European Union; LOD = limit of detection.

${ }^{2}$ No MRL.

${ }^{3}$ Not given.

antibiotics (Kumar et al., 2012). The use of G. stearothermophilus as an indicator might explain why some microbiological inhibition methods such as the Eclipse 100 (Beltrán et al., 2015) and BRT AiM (Molina et al., 2003) are mainly sensitive to penicillins and cephalosporins and poorly sensitive to other antibiotics. However, some microbiological inhibition methods use sensitizers such as TMP and CAP to improve the sensitivity of G. stearothermophilus to antibiotics, which greatly improves the sensitivity of the microbiological inhibition methods to sulfonamides, tetracyclines, and other antibiotics, including penicillins and cephalosporins (Nagel et al., 2012, 2013). Nevertheless, no single microbiological inhibition method can detect all types of antibiotics, including $\beta$-lactams, aminoglycosides, tetracyclines, macrolides, sulfonamides, and lincosamides in milk until now. Based on previous studies, we achieved some improvement toward optimization in this study. First, we improved the nutritional conditions of the kit's medium to promote the germination, growth, and acid production of the indicator bacteria in kit's medium. Second, sensitizers such as TMP and CAP were used to improve the sensitivity of test bacterium to antibiotics. Third, special additives (e.g., carboxymethylcellulose sodium) were added to the kit's medium to prevent interference from milk. Therefore, the kit in this study was sensitive not only to $\beta$-lactams, but also to tetracyclines, sulfonamides, lincosamides, most aminoglycosides, and most macrolides in milk.

The LOD of different microbiological inhibition methods for $\beta$-lactams, aminoglycosides, tetracyclines, macrolides, sulfonamides, and lincosamides in milk are given in Table 6 . The test kit was more sensitive to aminoglycosides than the BRT AiM (Molina et al., 2003), Copan milk test (Le Breton et al., 2007), Eclipse 100 (Montero et al., 2005), Delvotest SP-NT (Althaus et al., 2003a), and Charm Blue Yellow (Linage et al., 2007) tests. Moreover, the LOD of the test 
Table 4. The effects of different batches of medium on the sensitivity of the method to antibiotics

\begin{tabular}{|c|c|c|c|c|c|c|c|}
\hline \multirow[b]{2}{*}{ Antibiotic } & \multirow{2}{*}{$\begin{array}{l}\text { Spiked level } \\
(\cdot / \mathrm{L})\end{array}$} & \multicolumn{6}{|c|}{ Rate of positive results (\%) } \\
\hline & & 1 & 2 & 3 & 4 & 5 & Mean \\
\hline \multirow[t]{4}{*}{ Penicillin G } & 1 & 30 & 40 & 45 & 35 & 40 & 38 \\
\hline & 2 & 95 & 100 & 100 & 100 & 100 & 99 \\
\hline & 3 & 100 & 100 & 100 & 100 & 100 & 100 \\
\hline & 4 & 100 & 100 & 100 & 100 & 100 & 100 \\
\hline \multirow{5}{*}{ Ampicillin } & 1 & 0 & 0 & 0 & 0 & 0 & 0 \\
\hline & 2 & 85 & 100 & 95 & 95 & 90 & 93 \\
\hline & 3 & 100 & 100 & 100 & 100 & 100 & 100 \\
\hline & 4 & 100 & 100 & 100 & 100 & 100 & 100 \\
\hline & 10 & 100 & 100 & 100 & 100 & 100 & 100 \\
\hline \multirow{5}{*}{ Amoxicillin } & 1 & 0 & 0 & 0 & 0 & 0 & 0 \\
\hline & 2 & 100 & 90 & 95 & 95 & 95 & 95 \\
\hline & 3 & 100 & 100 & 100 & 100 & 100 & 100 \\
\hline & 4 & 100 & 100 & 100 & 100 & 100 & 100 \\
\hline & 10 & 100 & 100 & 100 & 100 & 100 & 100 \\
\hline \multirow{4}{*}{ Cloxacillin } & 10 & 30 & 30 & 25 & 20 & 25 & 26 \\
\hline & 15 & 100 & 95 & 95 & 90 & 90 & 94 \\
\hline & 20 & 100 & 100 & 100 & 100 & 100 & 100 \\
\hline & 30 & 100 & 100 & 100 & 100 & 100 & 100 \\
\hline \multirow{4}{*}{ Dicloxacillin } & 10 & 25 & 30 & 30 & 25 & 30 & 28 \\
\hline & 15 & 90 & 95 & 95 & 100 & 95 & 95 \\
\hline & 20 & 100 & 100 & 100 & 100 & 100 & 100 \\
\hline & 30 & 100 & 100 & 100 & 100 & 100 & 100 \\
\hline \multirow[t]{4}{*}{ Oxacillin } & 10 & 85 & 90 & 80 & 85 & 90 & 86 \\
\hline & 15 & 100 & 90 & 95 & 95 & 100 & 96 \\
\hline & 20 & 100 & 100 & 100 & 100 & 100 & 100 \\
\hline & 30 & 100 & 100 & 100 & 100 & 100 & 100 \\
\hline \multirow{4}{*}{ Nafcillin } & 7 & 75 & 60 & 55 & 85 & 75 & 70 \\
\hline & 8 & 100 & 90 & 95 & 90 & 95 & 94 \\
\hline & 9 & 100 & 100 & 100 & 100 & 100 & 100 \\
\hline & 30 & 100 & 100 & 100 & 100 & 100 & 100 \\
\hline \multirow[t]{4}{*}{ Penicillin V } & 4 & 80 & 95 & 95 & 85 & 90 & 89 \\
\hline & 5 & 100 & 100 & 100 & 100 & 100 & 100 \\
\hline & 6 & 100 & 100 & 100 & 100 & 100 & 100 \\
\hline & 30 & 100 & 100 & 100 & 100 & 100 & 100 \\
\hline \multirow[t]{4}{*}{ Ceftiofur } & 40 & 60 & 60 & 50 & 65 & 55 & 58 \\
\hline & 60 & 100 & 100 & 90 & 100 & 90 & 96 \\
\hline & 80 & 100 & 100 & 100 & 100 & 100 & 100 \\
\hline & 100 & 100 & 100 & 100 & 100 & 100 & 100 \\
\hline Cefalexin & 10 & 80 & 100 & 95 & 90 & 90 & 91 \\
\hline & 20 & 100 & 100 & 100 & 100 & 95 & 99 \\
\hline & 40 & 100 & 100 & 100 & 100 & 100 & 100 \\
\hline & 100 & 100 & 100 & 100 & 100 & 100 & 100 \\
\hline Cefoperazone & 20 & 30 & 25 & 25 & 35 & 25 & 28 \\
\hline & 40 & 100 & 100 & 90 & 100 & 95 & 97 \\
\hline & 60 & 100 & 100 & 100 & 100 & 100 & 100 \\
\hline & 50 & 100 & 100 & 100 & 100 & 100 & 100 \\
\hline Cefazolin & 5 & 0 & 0 & 0 & 0 & 0 & 0 \\
\hline & 10 & 100 & 90 & 85 & 95 & 100 & 94 \\
\hline & 20 & 100 & 100 & 100 & 100 & 100 & 100 \\
\hline & 50 & 100 & 100 & 100 & 100 & 100 & 100 \\
\hline Cefuroxime & 20 & 45 & 40 & 55 & 45 & 50 & 47 \\
\hline & 40 & 100 & 90 & 95 & 85 & 100 & 94 \\
\hline & 60 & 100 & 100 & 100 & 100 & 100 & 100 \\
\hline & 50 & 100 & 100 & 100 & 100 & 100 & 100 \\
\hline Streptomycin & 200 & 80 & 85 & 80 & 75 & 70 & 78 \\
\hline & 250 & 100 & 100 & 90 & 90 & 95 & 95 \\
\hline & 500 & 100 & 100 & 100 & 100 & 100 & 100 \\
\hline Dihydrostreptomycin & 200 & 55 & 60 & 75 & 55 & 70 & 63 \\
\hline & 250 & 100 & 100 & 90 & 95 & 95 & 96 \\
\hline & 500 & 100 & 100 & 100 & 100 & 100 & 100 \\
\hline Gentamicin & 25 & 40 & 45 & 55 & 50 & 50 & 48 \\
\hline & 50 & 90 & 100 & 100 & 95 & 95 & 96 \\
\hline & 75 & 100 & 100 & 100 & 100 & 100 & 100 \\
\hline & 100 & 100 & 100 & 100 & 100 & 100 & 100 \\
\hline & 200 & 100 & 100 & 100 & 100 & 100 & 100 \\
\hline
\end{tabular}


Table 4 (Continued). The effects of different batches of medium on the sensitivity of the method to antibiotics

\begin{tabular}{|c|c|c|c|c|c|c|c|}
\hline \multirow[b]{2}{*}{ Antibiotic } & \multirow{2}{*}{$\begin{array}{l}\text { Spiked level } \\
(\cdot / \mathrm{L})\end{array}$} & \multicolumn{6}{|c|}{ Rate of positive results (\%) } \\
\hline & & 1 & 2 & 3 & 4 & 5 & Mear \\
\hline \multirow[t]{5}{*}{ Neomycin } & 25 & 60 & 55 & 55 & 50 & 60 & 56 \\
\hline & 50 & 100 & 100 & 90 & 90 & 85 & 93 \\
\hline & 75 & 100 & 100 & 100 & 100 & 100 & 100 \\
\hline & 500 & 100 & 100 & 100 & 100 & 100 & 100 \\
\hline & 1,500 & 100 & 100 & 100 & 100 & 100 & 100 \\
\hline \multirow[t]{3}{*}{ Kanamycin } & 1,000 & 90 & 85 & 95 & 90 & 95 & 91 \\
\hline & 1,250 & 100 & 95 & 95 & 100 & 100 & 98 \\
\hline & 1,500 & 100 & 100 & 100 & 100 & 100 & 100 \\
\hline \multirow[t]{3}{*}{ Spectinomycin } & 1,000 & 85 & 95 & 80 & 95 & 95 & 90 \\
\hline & 1,250 & 100 & 100 & 100 & 95 & 95 & 98 \\
\hline & 1,500 & 100 & 100 & 100 & 100 & 100 & 100 \\
\hline \multirow[t]{3}{*}{ Doxycycline } & 75 & 70 & 80 & 75 & 65 & 75 & 73 \\
\hline & 100 & 100 & 100 & 100 & 100 & 90 & 98 \\
\hline & 200 & 100 & 100 & 100 & 100 & 100 & 100 \\
\hline \multirow[t]{3}{*}{ Chlorotetracycline } & 75 & 60 & 65 & 70 & 70 & 75 & 68 \\
\hline & 100 & 90 & 90 & 80 & 100 & 100 & 92 \\
\hline & 200 & 100 & 100 & 100 & 100 & 100 & 100 \\
\hline \multirow[t]{3}{*}{ Tetracycline } & 75 & 80 & 75 & 80 & 70 & 75 & 76 \\
\hline & 100 & 90 & 95 & 95 & 100 & 100 & 96 \\
\hline & 200 & 100 & 100 & 100 & 100 & 100 & 100 \\
\hline \multirow[t]{3}{*}{ Oxytetracycline } & 75 & 55 & 60 & 50 & 60 & 55 & 56 \\
\hline & 100 & 85 & 90 & 95 & 100 & 95 & 93 \\
\hline & 200 & 100 & 100 & 100 & 100 & 100 & 100 \\
\hline \multirow[t]{3}{*}{ Erythromycin } & 30 & 10 & 10 & 15 & 15 & 10 & 12 \\
\hline & 40 & 85 & 90 & 95 & 95 & 100 & 93 \\
\hline & 50 & 100 & 100 & 100 & 100 & 100 & 100 \\
\hline \multirow{3}{*}{ Tylosin } & 40 & 60 & 85 & 90 & 75 & 80 & 78 \\
\hline & 50 & 100 & 95 & 100 & 95 & 95 & 97 \\
\hline & 75 & 100 & 100 & 100 & 100 & 100 & 100 \\
\hline \multirow[t]{3}{*}{ Tilmicosin } & 40 & 95 & 80 & 90 & 85 & 80 & 86 \\
\hline & 50 & 100 & 100 & 100 & 100 & 100 & 100 \\
\hline & 75 & 100 & 100 & 100 & 100 & 100 & 100 \\
\hline \multirow[t]{3}{*}{ Spiramycin } & 600 & 100 & 95 & 100 & 80 & 85 & 92 \\
\hline & 700 & 100 & 100 & 100 & 95 & 100 & 99 \\
\hline & 800 & 100 & 100 & 100 & 100 & 100 & 100 \\
\hline Sulfadimidine & 75 & 0 & 0 & 0 & 0 & 0 & 0 \\
\hline & 100 & 85 & 90 & 95 & 95 & 100 & 93 \\
\hline & 150 & 100 & 100 & 100 & 100 & 100 & 100 \\
\hline Sulfadiazine & 25 & 40 & 60 & 55 & 45 & 55 & 51 \\
\hline & 50 & 85 & 90 & 95 & 100 & 100 & 94 \\
\hline & 75 & 100 & 100 & 100 & 100 & 100 & 100 \\
\hline & 100 & 100 & 100 & 100 & 100 & 100 & 100 \\
\hline Sulfamethoxazole & 25 & 25 & 35 & 30 & 20 & 20 & 26 \\
\hline & 50 & 95 & 95 & 100 & 100 & 100 & 98 \\
\hline & 75 & 100 & 100 & 100 & 100 & 100 & 100 \\
\hline & 100 & 100 & 100 & 100 & 100 & 100 & 100 \\
\hline Sulfamonomethoxine & 25 & 55 & 40 & 45 & 35 & 45 & 44 \\
\hline & 50 & 100 & 100 & 100 & 100 & 100 & 100 \\
\hline & 75 & 100 & 100 & 100 & 100 & 100 & 100 \\
\hline & 100 & 100 & 100 & 100 & 100 & 100 & 100 \\
\hline Sulfamethoxypyridazine & 25 & 100 & 85 & 90 & 100 & 85 & 92 \\
\hline & 50 & 95 & 95 & 100 & 100 & 100 & 98 \\
\hline & 75 & 100 & 100 & 100 & 100 & 100 & 100 \\
\hline & 100 & 100 & 100 & 100 & 100 & 100 & 100 \\
\hline Sulfaquinoxaline & 75 & 65 & 75 & 70 & 60 & 55 & 65 \\
\hline & 100 & 95 & 100 & 100 & 100 & 100 & 99 \\
\hline & 150 & 100 & 100 & 100 & 100 & 100 & 100 \\
\hline Lincomycin & 150 & 100 & 90 & 85 & 95 & 80 & 90 \\
\hline & 200 & 90 & 95 & 100 & 100 & 100 & 97 \\
\hline & 300 & 100 & 100 & 100 & 100 & 100 & 100 \\
\hline
\end{tabular}


kit for GEN, NEO, STR, and DSTR antibiotics were less than or close to the MRL. The presence of calcium ions can improve the sensitivity of $G$. stearothermophilus to aminoglycosides; hence, the $\mathrm{CaCl}_{2}$ in the kit's medium strengthened the ability of the kit to detect aminoglycosides (Seymour et al., 1988). Furthermore, the kit was sensitive to tetracyclines at the MRL and more sensitive than the Copan milk test (Le Breton et al., 2007), Eclipse 100 (Beltrán et al., 2015), and BRT AiM (Molina et al., 2003). The Copan milk test (Le Breton et al., 2007), Eclipse 100 (Beltrán et al., 2015), and BRT AIM (Molina et al., 2003) are insensitive to tetracyclines for 3 main reasons. First, tetracyclines are broad-spectrum bacteriostatic drugs and their bacteriostatic ability against gram-positive bacterium is weak; however, the indicator G. stearothermophilus is a gram-positive bacterium (Tumini et al., 2015). Second, bivalent metal ions in milk such as $\mathrm{Ca}^{2+}$ can chelate tetracyclines, resulting in weaker bacteriostatic ability of tetracyclines (Kuang et al., 2009). Third, the bacteriostatic activity of tetracyclines is stronger in a slightly acidic environment than in an alkaline condition (Mirecki and Nikolić, 2016). Chloramphenicol is widely used as a sensitizer of tetracyclines in microbiological inhibition methods. In this study, we used CAP in the kit medium to improve the bacteriostatic effect of tetracyclines to G. stearothermophilus. Additionally, carboxymethylcellulose sodium in the kit was used to chelate bivalent metal ions in milk. Therefore, the kit developed in this study was sensitive to tetracyclines, even in the alkaline medium ( $\mathrm{pH} 7.8 \pm 0.1)$. The ability of the test kit to detect macrolides was similar to that of the Delvotest SP-NT (Althaus et al., 2003a) and Charm Blue Yellow (Linage et al., 2007) tests, and the kit was more sensitive to macrolides than the Copan milk test (Le Breton et al., 2007), Eclipse 100 (Montero et al., 2005), and BRT AiM (Molina et al., 2003) tests. However, the sensitivity of the kit to spiramycin needs to be improved. In future study, some sensitizers such as spiramycin in the kit may improve the sensitivity of the kit to spiramycin and other macrolides. Trimethoprim is a sensitizer of sulfonamides, and thus the kit with TMP was sensitive to sulfonamides. The sensitivity of the kit for sulfonamides was similar to that of the Delvotest SP-NT (Beltrán et al., 2015), Copan milk test (Le Breton et al., 2007), and Charm Blue Yellow (Romero et al., 2016). However, the test kit could detect more antibiotics in the sulfonamide class. The kit had higher sensitivity to sulfonamides than the Eclipse 100 (Montero et al., 2005) and BRT

Table 5. Results of the screening and quantification test in incurred milk, samples taken from animals treated with antibiotics according to the pre-slaughter medication data

\begin{tabular}{|c|c|c|c|c|}
\hline \multirow[b]{2}{*}{ Antibiotic } & \multirow[b]{2}{*}{ Withdraw time (h) } & \multicolumn{2}{|c|}{ Positive/Total samples ${ }^{1}$} & \multirow{2}{*}{$\begin{array}{c}\text { UPLC-MS/MS } \\
(\cdot / \mathrm{L})\end{array}$} \\
\hline & & This kit & Eclipse 50 & \\
\hline \multirow[t]{5}{*}{ Ampicillin } & Before administration & $0 / 3$ & $0 / 3$ & $-^{3}$ \\
\hline & 0 & $0 / 3$ & $0 / 3$ & - \\
\hline & 24 & $3 / 3$ & $3 / 3$ & $2.3-6.5$ \\
\hline & 48 & $0 / 3$ & $0 / 3$ & - \\
\hline & 72 & $0 / 3$ & $0 / 3$ & - \\
\hline \multirow[t]{5}{*}{ Gentamicin } & Before administration & $0 / 3$ & $0 / 3$ & - \\
\hline & 0 & $0 / 3$ & $0 / 3$ & - \\
\hline & 24 & $3 / 3$ & $1 / 3$ & $100-270$ \\
\hline & 48 & $0 / 3$ & $0 / 3$ & $20-40$ \\
\hline & 72 & $0 / 3$ & $0 / 3$ & - \\
\hline \multirow[t]{5}{*}{ Oxytetracycline } & Before administration & $0 / 3$ & $0 / 3$ & - \\
\hline & 0 & $0 / 3$ & $0 / 3$ & - \\
\hline & 24 & $3 / 3$ & $3 / 3$ & $200-300$ \\
\hline & 48 & $3 / 3$ & $3 / 3$ & $100-165$ \\
\hline & 72 & $0 / 3$ & $0 / 3$ & $25-50$ \\
\hline \multirow[t]{5}{*}{ Tylosin } & Before administration & $0 / 3$ & $0 / 3$ & - \\
\hline & 0 & $0 / 3$ & $0 / 3$ & - \\
\hline & 24 & $3 / 3$ & $2 / 3$ & $45-95$ \\
\hline & 48 & $0 / 3$ & $0 / 3$ & $15-30$ \\
\hline & 72 & $0 / 3$ & $0 / 3$ & - \\
\hline \multirow[t]{5}{*}{ Sulfadiazine } & Before administration & $0 / 3$ & $0 / 3$ & - \\
\hline & 0 & $0 / 3$ & $0 / 3$ & - \\
\hline & 24 & $3 / 3$ & $3 / 3$ & $56-110$ \\
\hline & 48 & $0 / 3$ & $0 / 3$ & $25-40$ \\
\hline & 72 & $0 / 3$ & $0 / 3$ & - \\
\hline
\end{tabular}

${ }^{1}$ The Eclipse 50 kit is from ZEU-Inmunotec (Zaragoza, Spain).

${ }^{2}$ Ultra-high-performance liquid chromatography-tandem MS.

${ }^{3}$ Not detected. 
Table 6. Comparison of detection capability (limit of detection, $\mu \mathrm{g} / \mathrm{L}$ ) for antibiotics among microbiological inhibition methods

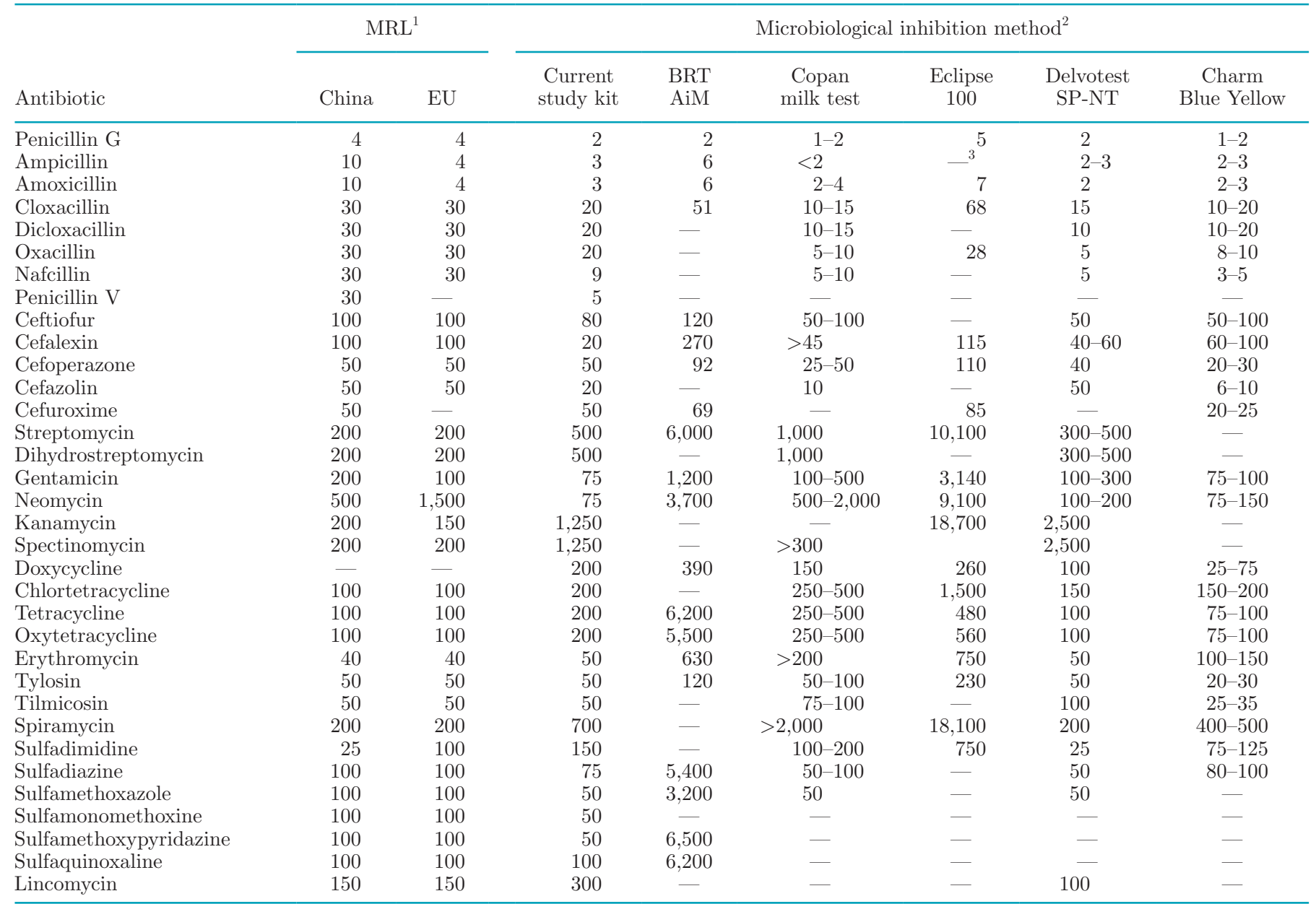

${ }^{1}$ Maxiumum residue limits (MRL) of China and the European Union.

${ }^{2}$ BRT AiM: AiM-Analytik in Milch Produktions-und Vertriebs GmbH (München, Germany); Copan milk test: Copan Italia SpA (Brescia, Italy); Eclipse 100: ZEU-Inmunotec SL (Zaragoza, Spain); Delvotest SP-NT: DSM Food Specialties (Delft, the Netherlands); Charm Blue Yellow II: Charm Sciences (Lawrence, MA).

${ }^{3}$ Not detected.

AiM (Molina et al., 2003) tests. The ability of this kit to detect $\beta$-lactams and lincosamides was improved by cross-reaction with the sensitizers of TMP and CAP. Thus, this kit was more sensitive to penicillins and cephalosporins than the Copan milk test (Le Breton et al., 2007), Eclipse 100 (Beltrán et al., 2015), and BRT AiM test (Molina et al., 2003). In addition to sensitizers, other components in the kit's medium such as $\mathrm{MnSO}_{4} \cdot \mathrm{H}_{2} \mathrm{O}, \mathrm{MgSO}_{4}$, glucose, and other nutrient substances can improve the detection capability of the kit. Manganese and magnesium ions are cofactors of key enzymes in glycolysis, promoting enzyme activity. Consequently, $\mathrm{MnSO}_{4} \cdot \mathrm{H}_{2} \mathrm{O}$ and $\mathrm{MgSO}_{4}$ improved the acid-producing ability of indicator bacteria and thus indirectly enhanced the sensitivity of the kit by lowering the concentration of indicator bacteria. However, a lower concentration of indicator bacteria in the medium results in a longer detection time. Nevertheless, the nutrients in the medium, including glucose, promote the germination, growth, and acid production of indicator bacteria, and thus shorten the detection time.

\section{Specificity}

Milk samples were directly added into the detection system without sample pretreatment other than heating in a water bath for $10 \mathrm{~min}$ at $80^{\circ} \mathrm{C}$. Antibiotics in milk can permeate into the detection medium during incubation. At the same time, natural bacteriostatic substances in milk, such as protein, fat (Andrew, 2000), somatic cells, other bacteria (Reybroeck et al., 2014), lactoferrin (Romero et al., 2014), lysozyme, and the lactoperoxidase system, can permeate into the detection medium (Althaus et al., 2003b). These natural 
bacteriostatic substances can inhibit the growth of $G$. stearothermophilus ATCC12980 in the detection system, which will interfere with the process of acid production and color change of the $\mathrm{pH}$ indicator, resulting in false-positive results. Moreover, some substances in milk might interfere with the antibacterial activity of antibiotics. For example, calcium ion in milk can chelate with tetracyclines, which results in false-negative results for tetracyclines. Additionally, a lot of protein in the milk permeates into the medium during the incubation process, and the white lactoprotein could obscure the color change of the $\mathrm{pH}$ indicator, making visual detection difficult.

Some microbiological methods such as BRT AiM (Molina et al., 2003) and Eclipse 100 (Montero et al., 2005) used a sample pretreatment of pre-permeation at $4^{\circ} \mathrm{C}$ for $1 \mathrm{~h}$. Compared with no pretreatment, the color change of the medium using the prediffusion method is clearer and the result is more accurate. However, the pre-permeation method at $4^{\circ} \mathrm{C}$ for $1 \mathrm{~h}$ prolonges the process time of the kit. Furthermore, the solvent extraction approach used in Premi Test, a commercial microbiological kit (DSM Food Specialties R\&D, Delft, the Netherlands), can significantly improve the detection ability of the microbiological inhibition method for antibiotics. However, the solvent extraction approach is laborious, time consuming, and only applicable for use in a laboratory (Stead et al., 2004).

In the present study, milk samples were incubated in a water bath for $10 \mathrm{~min}$ at $80^{\circ} \mathrm{C}$ before being added to the kit's medium; incubation at $80^{\circ} \mathrm{C}$ can inactivate natural bacteriostatic substances in milk and reduce the falsepositive rate. Consequently, the false-positive rate of this kit was low (1\%). The same pretreatment method was used in Houali et al. (2013). Moreover, heating can inactivate $\beta$-lactamases that may be present in milk, which prevents inactivation of the antibacterial activity of $\beta$-lactams present in the sample, and thus avoids the false-negative results for $\beta$-lactams. Additionally, carboxymethylcellulose sodium in the kit can chelate with calcium ion in milk, which prevents interference from calcium ions in milk and the false-negative results for tetracyclines. Therefore, the false-negative rate of this kit was $0 \%$. Furthermore, the carboxymethylcellulose sodium forms a complex network that can prevent macromolecular substances in milk such as protein and fat from permeating the kit's medium, which prevents the white lactoprotein from obscuring the color change of the $\mathrm{pH}$ indicator. Accordingly, it is easy to observe results of the color change at the end of detection. In summary, the sample pretreatment method in this study was easy to conduct, timesaving, and cost effective, and could be applied anywhere.

\section{Stability}

During the production of this kit, new materials and new production technology were used, and the culture medium of the kit was further improved to ensure a long shelf life. The detection medium used in this kit, the auxiliary ingredients, microplates, aluminized film cover, and other materials all underwent an aseptic treatment. To perform the test, $150 \mu \mathrm{L}$ of the culture medium was added into individual wells of microtiter plates using an electronic pipette under sterile conditions. Then, the disposable aluminum platinum cover was used to seal the wells in each microtiter plate for storage at $4^{\circ} \mathrm{C}$ until use. Therefore, water in the detection medium is not lost, and the bacteria and $\mathrm{CO}_{2}$ in the outside environment cannot enter and contaminate the kit's medium. Moreover, the carboxymethylcellulose sodium and polyethylene glycol 4000 can bind with water by hydrogen bonding, which further prevents water in the kit's medium from evaporating. The acidproducing ability of G. stearothermophilus ATCC12980 spores in the kit and their sensitivity to antimicrobial agents remained unchanged for months. Consequently, the kit developed in the present study is stable and the shelf life is more than 6 mo.

\section{CONCLUSIONS}

We developed a microbiological inhibition method that can detect 34 common antibiotic residues in milk, including those in the $\beta$-lactam, aminoglycoside, tetracycline, sulfonamide, macrolide, and lincosamide classes. The LOD of the kit for $\beta$-lactams, aminoglycosides (GEN, NEO), macrolides (TYL, TIL), and sulfonamides were less than or equal to the MRL determined by China and the EU. The LOD for tetracyclines, STR, DSTR, KAN, SPE, ERY, SPI, SDM, and LIN were higher than the MRL established by China and the EU. Moreover, the system was more sensitive to some antibiotics in milk, such as aminoglycosides, than other currently used microbiological methods. Validation and comparison of this microbiological system and other commercially available microbiological methods to detect antimicrobials in ewe and goat milks should be performed. Then, this microbiological inhibition method can be commercialized based on validation test and optimization.

\section{ACKNOWLEDGMENTS}

This work was supported by 2019 National Risk Assessment of Quality and Safety of Agricultural Product 
(GJFP2019027) and the Key Project of the Ministry of Agriculture (2011-G5, Beijing, China).

\section{REFERENCES}

Althaus, R., A. Torres, C. Peris, M. C. Beltran, N. Fernandez, and M. P. Molina. 2003a. Accuracy of BRT and Delvotest microbial inhibition tests as affected by composition of ewe's milk. J. Food Prot. 66:473-478. https://doi.org/10.4315/0362-028X-66.3.473.

Althaus, R. L., A. Torres, A. Montero, S. Balasch, and M. P. Molina. 2003b. Detection limits of antimicrobials in ewe milk by Delvotest photometric measurements. J. Dairy Sci. 86:457-463. https://doi .org/10.3168/jds.S0022-0302(03)73624-8.

Andrew, S. M. 2000. Effect of fat and protein content of milk from individual cows on the specificity rates of antibiotic residue screening tests. J. Dairy Sci. 83:2992-2997. https://doi.org/10.3168/jds .S0022-0302(00)75200-3.

Baur, D., B. P. Gladstone, F. Burkert, E. Carrara, F. Foschi, S. Döbele, and E. Tacconelli. 2017. Effect of antibiotic stewardship on the incidence of infection and colonisation with antibiotic-resistant bacteria and Clostridium difficile infection: A systematic review and meta-analysis. Lancet Infect. Dis. 17:990-1001. https://doi .org/10.1016/S1473-3099(17)30325-0.

Beltrán, M. C., M. I. Berruga, A. Molina, R. L. Althaus, and M. P. Molina. 2015. Performance of current microbial tests for screening antibiotics in sheep and goat milk. Int. Dairy J. 41:13-15. https:/ /doi.org/10.1016/j.idairyj.2014.09.007.

Berruga, M. I., A. Molina, R. L. Althaus, and M. P. Molina. 2016. Control and prevention of antibiotic residues and contaminants in sheep and goat's milk. Small Rumin. Res. 142:38-43. https://doi .org/10.1016/j.smallrumres.2016.02.023.

Breen, J., A. Bradley, P. Davies, P. Down, K. Fitzgerald, C. Hudson, J. Huxley, R. Hyde, J. Remnant, W. Wapenaar, M. Green, S.-J. Redman, W. McCarthy, R. Cavill, and G. McPherson. 2017. Clinical forum: The responsible use of antimicrobial therapy in the control of clinical mastitis and somatic cell count in dairy herds. Livestock (Lond) 22:290-296. https://doi.org/10.12968/live.2017.22.6.290.

Choma, I. M., E. M. Grzelak, and B. Majer-Dziedzic. 2012. Comparison of deproteinization methods used before TLC-DB and HPLC analysis of flumequine residues in milk. Med. Chem. 8:95-101. https://doi.org/10.2174/157340612799278423.

Chu, L., J. Deng, and X. Kang. 2017. Packed-nano fiber solid phase extraction coupled with HPLC for the determination of chloramphenicol in milk. Anal. Methods 9:6499-6506. https://doi.org/10 $.1039 / \mathrm{c} 7 \mathrm{ay} 01192 \mathrm{j}$.

Darwish, W. S., E. A. Eldaly, M. T. El-Abbasy, Y. Ikenaka, S. Nakayama, and M. Ishizuka. 2013. Antibiotic residues in food: The African scenario. Jpn. J. Vet. Res. 61. https://doi.org/10.14943/ jjvr.61.suppl.s13.

de Albuquerque Fernandes, S. A., A. P. A. Magnavita, S. P. B. Ferrao, S. A. Gualberto, A. S. Faleiro, A. J. Figueiredo, and S. V. Matarazzo. 2014. Daily ingestion of tetracycline residue present in pasteurized milk: A public health problem. Environ. Sci. Pollut. Res. Int. 21:3427-3434. https://doi.org/10.1007/s11356-013-2286-5.

Debayle, D., G. Dessalces, and M. F. Grenier-Loustalot. 2008. Multiresidue analysis of traces of pesticides and antibiotics in honey by HPLC-MS-MS. Anal. Bioanal. Chem. 391:1011-1020. https://doi .org/10.1007/s00216-008-2003-2.

European Union. 2010. Commission Regulation (EU) No 37/2010 of 22 December 2009 on pharmacologically active substances and their classification regarding maximum residue limits in foodstuffs of animal origin. Off. J. L15:1-72.

Ezenduka, E. V., O. S. Ike, and N. J. Anaelom. 2014. Rapid detection of antimicrobial residues in poultry: A consequence of non-prudent use of antimicrobials. Health 6:149-152. https://doi.org/10.4236/ health.2014.62023.

FAO/WHO (Food and Agricultures of the United Nations/World Health Organization). 2015. Maximum Residue Limits (MRLs) and Risk Management Recommendations (RMRs) for residues of veterinary drugs in foods CAC/MRL 2-2015 1-41. http://www .codexalimentarius.org/standards/list-of-standards/en/?provide $=$ standards\&orderField $=$ fullReference\&sort $=$ asc \&num $1=\mathrm{CAC} /$ MRL.

Hakimzadegan, M., M. K. Khosroshahi, and S. H. Nasab. 2014. Monitoring of antibiotic residue in chicken eggs in Tabriz city by FPT Int. J. Adv. Biol. Biomed. Res. 2:132-140.

Houali, K., B. Yabrir, O. Malki, A. Chergui, N. Chenouf, S. Yahiaoui, M. Labiad, H. Ghenim, and V. M. Cluj-Napoca. 2013. Detection of antibiotics residues in raw milk produced in Freha Area (TiziOuzou), Algeria. JOURNAL NAME 70:83-87.

ISO. (International Organization for Standardization). 2003. Milk and milk products - Guidelines for a standardized description of microbial inhibition tests. ISO13969:2003/IDF 183:2003. ISO, Geneva, Switzerland.

Jank, L., M. T. Martins, J. B. Arsand, T. M. C. Motta, T. C. Feijó, T. dos Santos Castilhos, R. B. Hoff, F. Barreto, and T. M. Pizzolato. 2017. Liquid chromatography-tandem mass spectrometry multiclass method for 46 antibiotics residues in milk and meat: Development and validation. Food Anal. Methods 10:2152-2164. https://doi.org/10.1007/s12161-016-0755-4

Kuang, Y., H. Jia, K. Miyanaga, and Y. Tanji. 2009. Effect of milk on antibacterial activity of tetracycline against Escherichia coli and Staphylococcus aureus isolated from bovine mastitis. Appl. Microbiol. Biotechnol. 84:135-142. https://doi.org/10.1007/s00253-009 $-2008-6$.

Kumar, N., H. V. Raghu, A. Kumar, L. Haldar, A. Khan, S. Rane, and R. K. Malik. 2012. Spore germination based assay for monitoring antibiotic residues in milk at dairy farm. World J. Microbiol. Biotechnol. 28:2559-2566. https://doi.org/10.1007/s11274-012-1065 -7 .

Le Breton, M. H., M. C. Savoy-Perroud, and J. M. Diserens. 2007. Validation and comparison of the Copan Milk Test and Delvotest SP-NT for the detection of antimicrobials in milk. Anal. Chim. Acta 586:280-283. https://doi.org/10.1016/j.aca.2006.11.060.

Linage, B., C. Gonzalo, J. A. Carriedo, J. A. Asensio, M. A. Blanco, L. F. De La Fuente, and F. San Primitivo. 2007. Performance of blueyellow screening test for antimicrobial detection in ovine milk. J. Dairy Sci. 90:5374-5379. https://doi.org/10.3168/jds.2007-0245.

Mata, L., D. Sanz, and P. Razquin. 2014. Validation of the Explorer ${ }^{\circledR}$ 2.0 test coupled to e-Reader ${ }^{\circledR}$ for the screening of antimicrobials in muscle from different animal species. Food Addit. Contam. Part A Chem. Anal. Control Expo. Risk Assess. 31:1496-1505. https:// doi.org/10.1080/19440049.2014.934303.

Mirecki, S., and N. Nikolić. 2016. Influence of preservative concentration, $\mathrm{pH}$ value and fat content in raw milk at detection limit of microbial inhibitor tests (Delvotest ${ }^{\circledR}$ Accelerator) for amoxicillin and oxytetracycline. Food Anal. Methods 9:2864-2871. https://doi .org/10.1007/s12161-016-0476-8.

Molina, M. P., R. L. Althaus, A. Molina, and N. Fernández. 2003. Antimicrobial agent detection in ewes' milk by the microbial inhibitor test brilliant black reduction test - BRT AiM ${ }^{\circledR}$. Int. Dairy J 13:821-826. https://doi.org/10.1016/S0958-6946(03)00107-9.

Montero, A., R. L. Althaus, A. Molina, I. Berruga, and M. P. Molina. 2005. Detection of antimicrobial agents by a specific microbiological method (Eclipse100 ${ }^{\circledR}$ ) for ewe milk. Small Rumin. Res. 57:229237. https://doi.org/10.1016/j.smallrumres.2004.07.006.

Mor, F., F. Sahindokuyucu Kocasari, G. Ozdemir, and B. Oz. 2012. Determination of sulphonamide residues in cattle meats by the Charm-II system and validation with high performance liquid chromatography with fluorescence detection. Food Chem. 134:1645-1649. https://doi.org/10.1016/j.foodchem.2012.03.049.

Nagel, O., M. P. Molina, and R. Althaus. 2013. Microbiological system in microtitre plates for detection and classification of antibiotic residues in milk. Int. Dairy J. 32:150-155. https://doi.org/10 .1016/j.idairyj.2013.04.004.

Nagel, O. G., M. C. Beltrán, M. P. Molina, and R. L. Althaus. 2012. Novel microbiological system for antibiotic detection in ovine milk. Small Rumin. Res. 102:26-31. https://doi.org/10.1016/j .smallrumres.2011.11.018.

Reybroeck, W., M. De Vleeschouwer, S. Marchand, D. Sinnaeve, K. Heylen, J. De Block, A. Madder, J. C. Martins, and M. Heyn- 
drickx. 2014. Cyclic lipodepsipeptides produced by Pseudomonas spp. naturally present in raw milk induce inhibitory effects on microbiological inhibitor assays for antibiotic residue screening. PLoS One 9. https://doi.org/10.1371/journal.pone.0098266.

Romero, T., M. C. Beltrán, I. Pérez-Baena, M. Rodríguez, and M. P. Molina. 2014. Effect of the presence of colostrum on microbial screening methods for antibiotic detection in goats' milk. Small Rumin. Res. 121:376-381. https://doi.org/10.1016/j.smallrumres 2014.07.007.

Romero, T., S. Van Weyenberg, M. P. Molina, and W. Reybroeck. 2016. Detection of antibiotics in goats' milk: Comparison of different commercial microbial inhibitor tests developed for the testing of cows' milk. Int. Dairy J. 62:39-42. https://doi.org/10.1016/j .idairyj.2016.07.004.

Seymour, E. H., G. M. Jones, and M. L. McGilliard. 1988. Comparisons of on-farm screening tests for detection of antibiotic residues. J. Dairy Sci. 71:539-544. https://doi.org/10.3168/jds.S0022 $-0302(88) 79587-9$

Stead, S., M. Sharman, J. A. Tarbin, E. Gibson, S. Richmond, J. Stark, and E. Geijp. 2004. Meeting maximum residue limits: An improved screening technique for the rapid detection of antimicrobial residues in animal food products. Food Addit. Contam. 21:216-221. https://doi.org/10.1080/02652030310001647280.

Steinborn, A., L. Alder, B. Michalski, P. Zomer, P. Bendig, S. A. Martinez, H. G. J. Mol, T. J. Class, and N. Costa Pinheiro. 2016. Determination of glyphosate levels in breast milk samples from germany by LC-MS/MS and GC-MS/MS. J. Agric. Food Chem. 64:1414-1421. https://doi.org/10.1021/acs.jafc.5b05852.

Tao, X., H. Jiang, J. Zhu, X. Wang, Z. Wang, L. Niu, X. Wu, W. Shi, and J. Shen. 2014. An ultrasensitive chemiluminescent ELISA for determination of chloramphenicol in milk, milk powder, honey, eggs and chicken muscle. Food Agric. Immunol. 25:137-148. https: //doi.org/10.1080/09540105.2012.753513.

Tian, H., J. Wang, Y. Zhang, S. Li, J. Jiang, D. Tao, and N. Zheng. 2016. Quantitative multiresidue analysis of antibiotics in milk and milk powder by ultra-performance liquid chromatography coupled to tandem quadrupole mass spectrometry. J. Chromatogr. B Analyt. Technol. Biomed. Life Sci. 1033-1034:172-179. https://doi .org/10.1016/j.jchromb.2016.08.023.

Tumini, M., O. G. Nagel, and R. L. Althaus. 2015. Microbiological bioassay using Bacillus pumilus to detect tetracycline in milk. J. Dairy Res. 82:248-255. https://doi.org/10.1017/S0022029915000138.

Wang, Y., X. Li, Z. Zhang, S. Ding, H. Jiang, J. Li, J. Shen, and X. Xia. 2016. Simultaneous determination of nitroimidazoles, benzimidazoles, and chloramphenicol components in bovine milk by ultra-high performance liquid chromatography-tandem mass spectrometry. Food Chem. 192:280-287. https://doi.org/10.1016/j .foodchem.2015.07.033.

Zeina, K., A. K. Pamela, and S. Fawwak. 2013. Quantification of antibiotic residues and determination of antimicrobial resistance profiles of microorganisms isolated from bovine milk in Lebanon. Food Nutr. Sci. 4:1-9. https://doi.org/10.4236/fns.2013.47A001. 